\title{
Relapse of tagraxofusp treated blastic plasmacytoid dendritic cell neoplasm with loss of CD123 expression
}

\author{
Rohit Gulati $^{1}$ (i) $\cdot$ Asma Abu-Salah ${ }^{1}$ (D) Tareq Salous $^{2}$ (D) $\cdot$ Mehdi Nassiri $^{1} \mathbb{C}$
}

Received: 23 September 2021 / Accepted: 15 November 2021 / Published online: 2 December 2021

(c) The Author(s) 2021

\begin{abstract}
Tagraxofusp, a CD123-based-targeted immunotherapy, was recently approved to treat blastic plasmacytoid dendritic cell neoplasm (BPDCN) with excellent response. Also, a subset of BPDCN shows resistance to tagraxofusp. These resistant cases continue to express CD123, which forms the basis of the continued utility of tagraxofusp in newer combination chemotherapies to overcome resistance in BPDCN. Herein, we report a case of an elderly male with BPDCN that achieved complete remission on initial primary treatment with tagraxofusp. However, BPDCN relapsed after 1.5 years while on treatment, with loss of CD123 expression. At relapse, the neoplasm was comprehensively immunophenotyped by flow cytometry (performed on both peripheral blood and bone marrow specimen) and by immunohistochemical evaluation of the bone marrow clot section. The neoplasm at relapse was diagnostic of BPDCN with a lack of CD123 expression. This case highlights a potential limitation of current and upcoming tagraxofusp-based multidrug therapies, at least in a subset of refractory BPDCN. We believe our report will serve as a sentinel to incite future investigations involving alternate resistance mechanisms in BDPCN.
\end{abstract}

Keywords SL-401 - Blastic plasmacytoid dendritic cell neoplasm · Diphtheria toxin - IL3 receptor alpha subunit · Tagraxofusp resistance $\cdot$ DT388 IL-3

\section{Introduction}

Targeted therapy has found a new success story through the growing utility of tagraxofusp, a CD123-directed antineoplastic drug. Tagraxofusp (SL-401, DT388IL-3, or tagraxofusp-erzs) internalizes into target cells by binding surface IL-3 receptor alpha (CD123) and inhibits cellular protein translocation subsequently causing cell death $[1,2]$. Blastic plasmacytoid dendritic cell neoplasm (BPDCN) is a

Rohit Gulati

rohitleo@gmail.com; rogulati@iu.edu

Asma Abu-Salah

asma.abusalah@gmail.com; askabusa@iu.edu

Tareq Salous

salous.tareq@gmail.com; tsalous@iu.edu

Mehdi Nassiri

mnassiri@iu.edu

1 Department of Pathology and Laboratory Medicine, Indiana University School of Medicine, 350 W 11th Street, Room 5048, Indianapolis, IN 46202, USA

2 Department of Medicine- Hematology/Oncology, 535 Barnhill Drive, Indianapolis, IN 46202, USA rare, aggressive neoplasm derived from the precursor plasmacytoid dendritic cells characterized by the expression of CD303, CD123, CD56 CD4, and TCL1, [3-5]. This ubiquitous expression of CD123 makes BPDCN a prime target for treatment with tagraxofusp, as described in a pilot study by Frankel et al. [6]. Subsequently, a multicenter clinical trial for single-agent tagraxofusp showed complete response in $72 \%$ cases on initial therapy and an overall response rate of $67 \%$ in relapsed refractory patients of BPDCN [7]. In response, the FDA approved tagraxofusp for the management of BPDCN in December 2018. The utility of this promising new drug extends potentially to other neoplasms strongly expressing CD123, including acute myeloid leukemia (AML) [8], hairy cell leukemia [9], and B-lymphoblastic leukemia [10]. The effectiveness of this drug has also been tested in tyrosine kinase-resistant CML [11].

Tagraxofusp resistance has been observed in a subset of cases in early clinical trials $[6,7]$ and published reports [12, 13]. Of interest, refractory BPDCN continues to express CD123 both during and after treatment, highlighting intact IL3-CD123-mediated binding-internalization of tagraxofusp in the target cell $[14,15]$. Utilizing the persistent targeting ability of tagraxofusp in CD123 expressing refractory 
BPDCN, combination therapy of tagraxofusp with hypomethylating agents (like azacitidine) has shown promising results to reverse diphtheria toxin (DT) resistance [14]. Herein, we report the first case (to the best of our knowledge) of tagraxofusp refractory BPDCN with no identifiable expression of CD123 on relapse. Our case warrants additional investigation towards a potential new pathway of resistance, and possibly, limited utility of the upcoming CD123-dependent multidrug regimen for treatment of refractory BPDCN.

\section{Clinical history}

A 78-year-old male has a long history of Crohn's disease (post ileectomy, not requiring continuous medications) and hypothyroidism (on levothyroxine). Seven years ago, the patient was diagnosed with a plasma cell neoplasm with monoclonal $\operatorname{IgG}$ lambda $(1.0 \mathrm{~g} / \mathrm{dL})$ protein, hemoglobin $(\mathrm{Hb})$ $10.9 \mathrm{~g} / \mathrm{dL}$, mean corpuscular volume (MCV) $102 \mathrm{fl}$, white blood cells (WBC) 2000/ $\mu \mathrm{L}$ (absolute neutrophil count (ANC): $700 / \mu \mathrm{L}$; absolute lymphocyte count (ALC) 1200k/ $\mu \mathrm{L}$ ), and platelets $172 \times 10^{3} / \mu \mathrm{L}$. The bone marrow at the time showed involvement by $20 \%$ clonal plasma cells with large clusters, with no bone lesions on the radiological survey. Karyotype showed 46,XY [20]. And the patient was treated with lenalidomide and dexamethasone followed by autologous stem cell transplant to achieve remission. After 6 years, the patient developed a new-onset skin rash. A bone marrow biopsy at this time showed extensive ( 85\%) involvement by blast-like population positive for CD4 (dim), CD56 (heterogenous) (flow cytometry), CD123, and TCL1 (immunohistochemistry (IHC)) and was diagnosed as BPDCN. The karyotype was normal 46,XY [20], and targeted molecular studies showed mutations in TET2 and ZRSR2. Treatment with tagraxofusp $(12 \mu \mathrm{g} / \mathrm{kg}$ once daily for five days in a 21-day cycle) resulted in the resolution of bone marrow involvement and skin lesions. The patient stayed in remission for 1.5 years followed by sudden onset leukocytosis. Physical examination now was unremarkable with no skin rash, lymphadenopathy, or organomegaly.

\section{Materials and methods}

Peripheral blood smear evaluation (WBC 16.4k/uL, Hg: $7.8 \mathrm{~g} / \mathrm{dL}$; MCV118; and platelets $80 \mathrm{k} / \mathrm{uL}$ ) revealed large immature appearing cells with a round to folded nucleus, vesicular chromatin, scant cytoplasm with rare cytoplasmic projections. Flow cytometry showed immature cells to be positive for dim CD45 expression and low side scatter, located in the "blast gate," and expressed CD56, CD117 (small subset), HLA-DR, CD7, and CD38, but negative for
CD123 (clone: Beckman Coulter ${ }^{\mathrm{TM}}$ SSDCLY107D2), CD34, myeloid/monocytic markers (myeloperoxidase (MPO), CD33, CD13, CD41, CD14, CD64) and lymphoid markers (surface and cytoplasmic CD3, and CD19) (Fig. 1). The bone marrow biopsy was 80-90\% cellular and extensively involved by sheets of large immature cells (Fig. 2B). Trilineage hematopoiesis was essentially absent. The aspirate smear showed frequent immature cells (Fig. 2A) that morphologically and immunophenotypically (flow cytometry) were similar to peripheral blood. Immunohistochemistry $(I H C)$ on the bone marrow clot section (Fig. 2C-E) additionally showed expression of TCL1 and confirmed the expression of CD56 and absence of CD123 (clone: 6H6, Cell Marque ${ }^{\mathrm{TM}}$ ), CD303 (BDCA-2), cytokeratin (cocktail), CD138, and CD68, MPO, CD3, and PAX-5 with adequately working controls. Karyotype at this time showed 45,XY,add(12)(p10),-20,+mar[1 2]/46,XY[8].

\section{Results}

These immunomorphological findings confirmed a relapse of BPDCN without expression of CD123.

\section{Discussion}

We report a relapse of BPDCN while on treatment with single-agent tagraxofusp $(12 \mathrm{uL} / \mathrm{kg})$, with no identifiable expression of CD123 as evaluated by two different methods (IHC and flow cytometry) and two different antibody clones. BPDCN is frequently diagnosed based on the coexpression of CD123, TCL1, CD56, and CD4 on neoplastic cells in the absence of other lineage-defining markers like MPO, CD19, and CD3. Although the absence of CD303 and $\mathrm{CD} 123$ expression in our case challenges a diagnosis of BPDCN and raises close differential, especially, with acute undifferentiated leukemia. The clinical history, characteristic morphology, and co-expression of CD56 and TCL1 (strong and nuclear) in the absence of markers defining other lineages confirmed the diagnosis [16, 17]. We also highlight that while CD303 has a very high diagnostic specificity for BPDCN, its utility may somewhat be limited by suboptimal sensitivity ( 70\%) [4]. In addition, flow cytometric findings were concordant on both peripheral blood and bone marrow specimen collected 2 days apart. The lack of identifiable expression of CD123 may potentially be explained by downregulation or internalization of receptor components. Also, a potential block of CD123 (IL3 receptor) by tagraxofusp causing a failure of identification is theoretically possible. However, it has not yet been reported even during therapy.

Tagraxofusp, recombinant IL3+ truncated (catalytic and translocation domain of DT), acts via a two-step process. 
Fig. 1 Dot plots for flow cytometric analysis of peripheral blood at relapse of BPDCN. The population of BPDCN cells is highlighted in red color and involves the "blast gate" with low side scatter and dim CD45 expression (A). Relapsed BPDCN is negative for CD123 (B) and shows CD56 expression $(\mathbf{C})$. BPDCN cells show no expression of CD11b or CD64 (D)
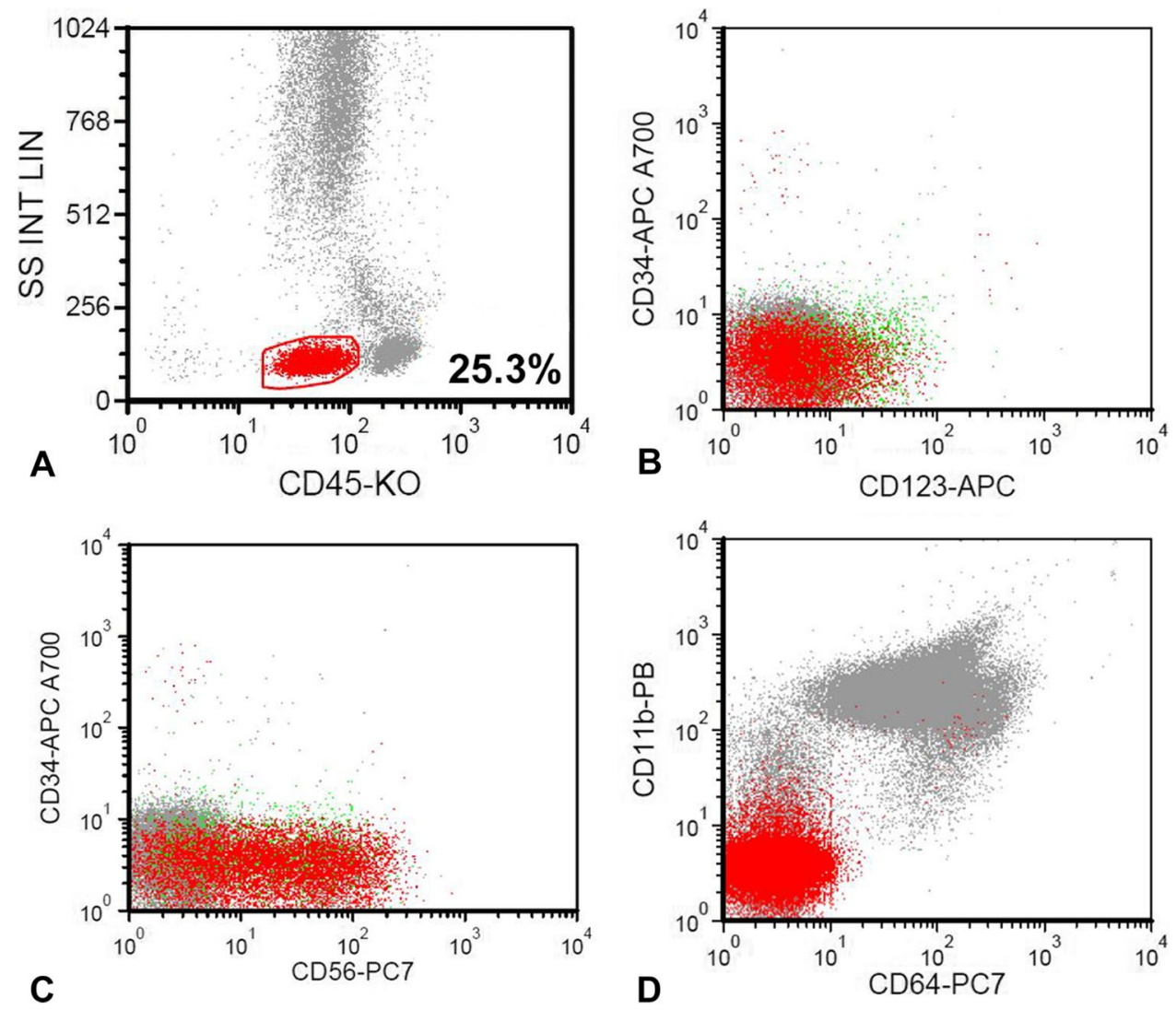

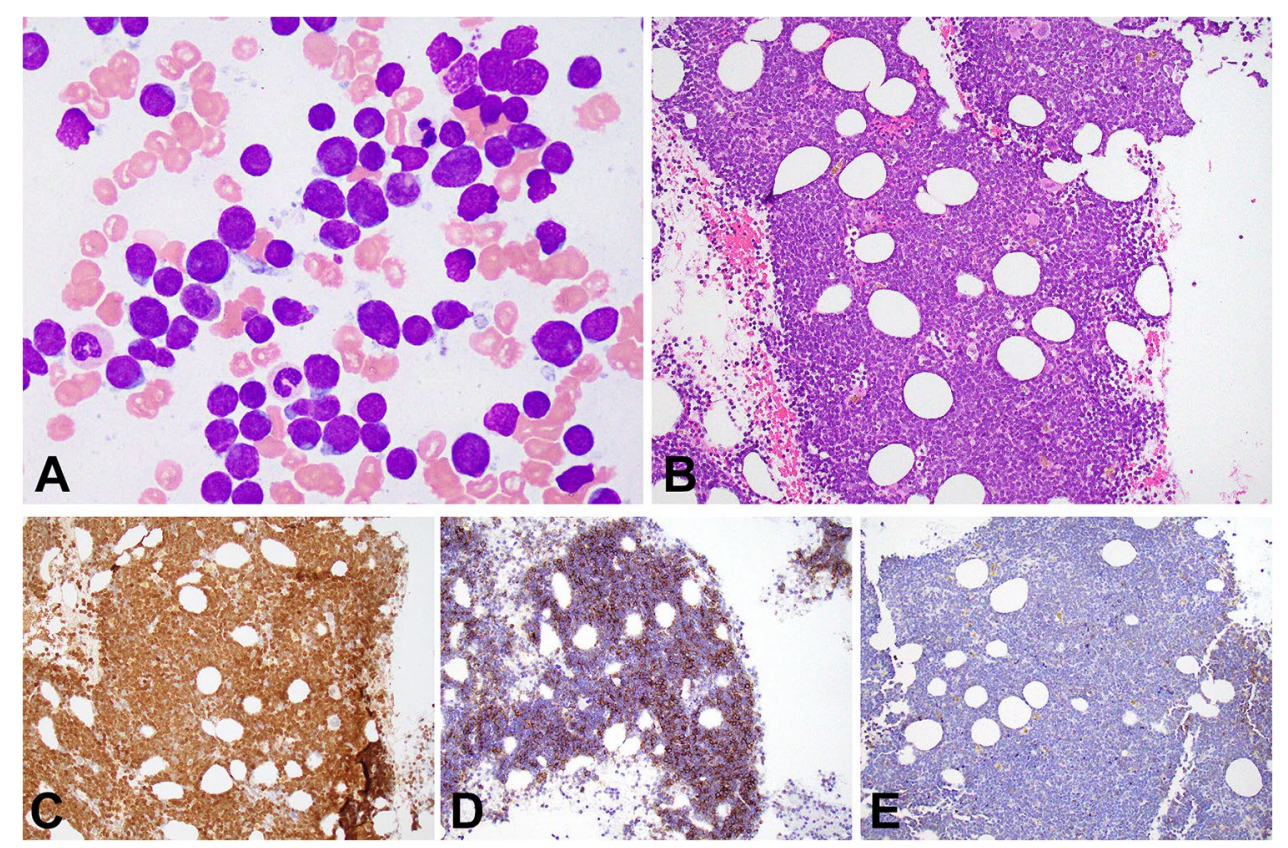

Fig. 2 Bone marrow evaluation at relapse of blastic plasmacytoid dendritic cell neoplasm (BPDCN). Fig.2A shows bone marrow aspirate (Wright-Giemsa Fuccillo stain modification; original magnification $\times 1000$ ) with frequent BPDCN cells characterized by large immature cells with round to folded nuclear contours, and high nuclear to cytoplasmic ratio and rare with cytoplasmic projections. Fig. 2B shows bone marrow clot section (hematoxylin-eosin stain; original magnification $\times 200$ ) with a hypercellular marrow showing extensive involvement by sheets of atypical large mononuclear immature BPDCN cells. Panels $\mathbf{C}, \mathbf{D}, \mathbf{E}$ show immunohistochemical staining with original magnification $\times 200$ of BPDCN on treatment with tagraxofusp showing positive expression of TCL1 (strong) (C), CD56 (D), and no expression of CD123 (E) 
First, it involves IL3 mediated binding/internalization to target the CD123 [IL-3(r)-alpha] positive tumor cells. The second step involves the cytoplasmic localization of the truncated DT to cause ADP phosphorylation of the histidine 715 on eEF2, causing a block of protein synthesis and eventually cell death. The resistance to tagraxofusp was evident in a subset of cases from early clinical trials. While no acquired somatic mutations were identified by whole exome sequencing, the resistance correlated with an acquired downregulation of $\mathrm{DPHI}$ (diphthamide biosynthesis 1) in the tagraxofusp resistant clones $[14,15]$. DPH1, the first enzyme in a series of at least seven member enzymes, converts histidine 715 on eEF2 to diphthamide [18], resulting in loss of direct target of DT mediated inhibition of translocation, i.e., DT resistance.

Togami et al. elegantly describe the persistent expression of CD123 during and after treatment in BPDCN and AML to be associated with a survival advantage. Also, they highlight relative dependency on CD123 for survival in AML cell lines compared to other disease types, based on a geneknockout experiment on cell lines from the genome-wide RNA interference screening [19,20]. Thus, the absence of CD123 expression in myeloid lineage cancers, AML (and BPDCN) would be disadvantageous for the survival of the CD123 negative clone, especially during treatment.

Similarly, BPDCN resistance to tagraxofusp demonstrated by other authors has shown persistent surface expression of CD123. In their series of pediatric BPDCN, Sun et al. described tagraxofusp resistance in one case with multiple relapses with CD123 positive neoplastic cells after two rounds of therapy. Two other cases showed good initial response followed by disease progression. One of these (tested) cases was positive for CD123 at disease progression [12]. More recently, Samhouri et al. described a case of an elderly male with BPDCN (involving skin) and concomitant myelodysplastic syndrome with ring sideroblasts (bone marrow) as refractory to low dose tagraxofusp $(5 \mu \mathrm{g} / \mathrm{kg}$; due to drug intolerance) and eventually achieved remission after switching to combination venetoclax and azacytidine [13].

Multidrug therapy has been used to overcome singleagent tagraxofusp resistance. Togami et al. confirmed the regulation of $D P H 1$ by reversible promoter CpG DNA methylation, as seen previously [21], in AML and BPDCN. They showed reversal of DT-based resistance by synergistic cytotoxicity of CD123-mediated targeting by tagraxofusp with the hypomethylating effect of azacitidine in patientderived xenografts models. Secondly, tagraxofusp resistance in both AML and BPDCN was associated with increased overall apoptotic priming and showed increased dependence on BCL-2 and or BCL-XL [15]. Such results have led to multiple trials evaluating combinations of tagraxofusp, azacitidine, and venetoclax (BCL-2 inhibitor) in BPDCN \{NCT03113643, NCT03485547, and NCT04216524\} Along similar lines, success stories of combination tagraxofusp and azacitidine in the management of relapsed BPDCN [22] highlight bright scope for specific multidrug management in BPDCN.

\section{Conclusion}

Herein, we describe an intriguing first example of tagraxofusp refractory BPDCN that relapsed while on full-dose single-agent tagraxofusp with loss of CD123 expression as evaluated by different methods. Although additional studies are required to understand the mechanism behind the absence of identifiable expression of CD123, our case may highlight a potential limitation of targeting CD123 in a subset of refractory BPDCN. We believe our report will be of significance to the current scientific literature on CD123based-targeted immunotherapy and should induce a formal investigation into alternative mechanisms of BPDCN resistance and its resolution.

Abbreviations BPDCN: blastic plasmacytoid dendritic cell neoplasm; eEF-2: eukaryotic elongation factor-2; AML: Acute myeloid leukemia; DT: diphtheria toxin; Hb: hemoglobin; MCV: mean corpuscular volume; WBC: white blood cells; ANC: absolute neutrophil count; ALC: absolute lymphocyte count; IHC: immunohistochemistry; MPO: myeloperoxidase; $D P H 1$ : diphthamide biosynthesis 1

Author contribution RG originally diagnosed, conceptualized, wrote manuscript, and edited the final manuscript. AA performed diagnostic data curation and partially wrote the manuscript. TS reviewed and edited the clinical data in the manuscript. MN edited and curated the draft. All authors have read and approved the final manuscript.

Data availability Data sharing is not applicable to this article as no datasets were generated or analyzed during the current study.

\section{Declarations}

Ethics approval This case report was exempted (waived) by the institutional review board (IRB) at Indiana University School of Medicine (Protocol \# 11756).

Competing interests The authors declare no competing interests.

Open Access This article is licensed under a Creative Commons Attribution 4.0 International License, which permits use, sharing, adaptation, distribution and reproduction in any medium or format, as long as you give appropriate credit to the original author(s) and the source, provide a link to the Creative Commons licence, and indicate if changes were made. The images or other third party material in this article are included in the article's Creative Commons licence, unless indicated otherwise in a credit line to the material. If material is not included in the article's Creative Commons licence and your intended use is not permitted by statutory regulation or exceeds the permitted use, you will 
need to obtain permission directly from the copyright holder. To view a copy of this licence, visit http://creativecommons.org/licenses/by/4.0/.

\section{References}

1. FitzGerald DJ (2014) Targeted diphtheria toxin to treat BPDCN. Blood 124(3):310-312

2. Frankel AE et al (2002) Diphtheria fusion protein therapy of chemoresistant malignancies. Curr Cancer Drug Targets 2(1):19-36

3. Chaperot $\mathrm{L}$ et al (2001) Identification of a leukemic counterpart of the plasmacytoid dendritic cells. Blood 97(10):3210-3217

4. Garnache-Ottou F et al (2009) Extended diagnostic criteria for plasmacytoid dendritic cell leukaemia. Br J Haematol 145(5):624-636

5. Khoury JD (2018) Blastic plasmacytoid dendritic cell neoplasm. Curr Hematol Malignan Rep 13(6):477-483

6. Frankel AE et al (2014) Activity of SL-401, a targeted therapy directed to interleukin-3 receptor, in blastic plasmacytoid dendritic cell neoplasm patients. Blood 124(3):385-392

7. Pemmaraju N et al (2019) Tagraxofusp in plastic plasmacytoid dendritic-cell neoplasm. N Engl J Med 380(17):1628-1637

8. Jordan CT et al (2000) The interleukin-3 receptor alpha chain is a unique marker for human acute myelogenous leukemia stem cells. Leukemia 14(10):1777-1784

9. Del Giudice I et al (2004) The diagnostic value of CD123 in B-cell disorders with hairy or villous lymphocytes. Haematologica 89(3):303-308

10. Muñoz L et al (2001) Interleukin-3 receptor alpha chain (CD123) is widely expressed in hematologic malignancies. Haematologica 86(12):1261-1269

11. Frolova O et al (2014) SL-401 and SL-501, targeted therapeutics directed at the interleukin-3 receptor, inhibit the growth of leukaemic cells and stem cells in advanced phase chronic myeloid leukaemia. Br J Haematol 166(6):862-874

12. Sun W et al (2018) First pediatric experience of SL-401, a CD123targeted therapy, in patients with blastic plasmacytoid dendritic cell neoplasm: report of three cases. J Hematol Oncol 11(1):61
13. Samhouri Y et al (2021) Tagraxofusp followed by combined azacitidine and venetoclax in blastic plasmacytoid dendritic cell neoplasm: a case report and literature review. J Oncol Pharm Pract 27(4):990-995

14. Togami K et al (2019) DNA methyltransferase inhibition overcomes diphthamide pathway deficiencies underlying CD123targeted treatment resistance. J Clin Invest 129(11):5005-5019

15. Stephansky J et al (2017) Resistance to SL-401 in AML and BPDCN Is associated with loss of the diphthamide synthesis pathway enzyme DPH1 and is reversible by azacitidine. Blood 130(Suppl_1):797-797

16. Alayed K et al (2013) TET2 mutations, myelodysplastic features, and a distinct immunoprofile characterize blastic plasmacytoid dendritic cell neoplasm in the bone marrow. Am J Hematol 88(12):1055-1061

17. Herling $\mathrm{M}$ et al (2003) TCL1 expression in plasmacytoid dendritic cells (DC2s) and the related CD4+ CD56+ blastic tumors of skin. Blood 101(12):5007-5009

18. Su X, Lin Z, Lin H (2013) The biosynthesis and biological function of diphthamide. Crit Rev Biochem Mol Biol 48(6):515-521

19. Tsherniak A et al (2017) Defining a cancer dependency map. Cell 170(3):564-576.e16

20. Ceribelli $\mathrm{M}$ et al (2016) A druggable TCF4- and BRD4-dependent transcriptional network sustains malignancy in blastic plasmacytoid dendritic cell neoplasm. Cancer Cell 30(5):764-778

21. Hu X et al (2013) Methylation of the DPH1 promoter causes immunotoxin resistance in acute lymphoblastic leukemia cell line KOPN-8. Leuk Res 37(11):1551-1556

22. Wang SY et al (2021) Combination of tagraxofusp and azacitidine is an effective option for relapsed blastic plasmacytoid dendritic cell neoplasm after allogeneic hematopoietic stem-cell transplantation. Clin Lymphoma Myeloma Leuk

Publisher's note Springer Nature remains neutral with regard to jurisdictional claims in published maps and institutional affiliations. 